\title{
From Point A to MRI-Guided Brachytherapy in Cancer Cervix: Rationalizing Brachytherapy Practice in India
}

\author{
Sajal Goel ${ }^{1} \quad$ Munish Gairola ${ }^{2} \quad$ Anil K. Dhull ${ }^{3} \quad$ Vikas Roshan $^{4} \quad$ Meenakshi Mittal $^{5}$ \\ ${ }^{1}$ Department of Radiation Oncology, Patel Hospital, Jalandhar, \\ Punjab, India \\ 2Department of Radiation Oncology, Rajiv Gandhi Cancer Institute \\ and Research Center, Rohini, Delhi, India \\ ${ }^{3}$ Department of Radiation Oncology, Pt. B.D. Sharma PGIMS, \\ Rohtak, Haryana, India \\ ${ }^{4}$ Department of Radiation Oncology, Shri Mata Vaishno Devi \\ Narayana Superspeciality Hospital, Jammu and Kashmir, India \\ ${ }^{5}$ Department of Radiation Oncology, Ivy Hospital, Chandigarh, India \\ Asian J Oncol 2019;5:97-98
}

Cervical brachytherapy practice in India is diverse. The choice of appropriate technique depends on residual tumor topography at brachytherapy, availability of applicators, and expertise.

Image-based interstitial brachytherapy adequately covers parametrial and posterior disease, prevents overdosage to critical organs in small cervix, and spares sigmoid colon better. ${ }^{1}$ The residual tumor in pelvis should be thoroughly assessed using clinical examination, X-ray, computed tomography (CT) scan, magnetic resonance imaging (MRI), or ultrasound. Most radiation oncologists are comfortably performing CT-based brachytherapy. MRI-based brachytherapy is less commonly used. CT overestimates the disease but MRI is more conformal, although the latter requires more time and expertise. Ultrasound is an effective alternative to clinical examination and MRI of pelvis for delineating residual disease. ${ }^{2}$ Intraoperative ultrasound helps in guiding placement of uterine tandem and prevents uterine perforation. ${ }^{3}$ The structures chiefly contoured are gross tumor volume (GTV), high risk clinical target volume (HRCTV), intermediate risk clinical target volume (IRCTV), bladder, rectum, and sigmoid. ${ }^{4}$ The prescription points are Point A and HRCTV. ${ }^{4}$

The applicators used routinely are Fletcher Suit Delclos, Manchester Tandem and Ovoid, Ring and Tandem (including MRI compatible), Vienna, Martinez Universal Perineal Interstitial Template (MUPIT), and Syed Neblett template. ${ }^{5}$

MRI-based brachytherapy as routine seems difficult due to the uncommon practice of delineating IRCTV, high patient load, and cost. However, existing literature substantiates that MRI-based brachytherapy offers better local control, progression-free survival, overall survival, and less grade 3-4 complications of organs at risk. ${ }^{6,7}$ These advantages translate into less overall treatment cost. ${ }^{8}$ It seems a good alternative for future brachytherapists.

Note

The study was presented at: Proceedings from a multi-instipanel discussion in North Zone AROICON 2019 Organization held at Sri Guru Ram Das University of Medical Sciences, Amritsar, Punjab, on September 21, 2019.

\section{Funding}

None.

\section{Conflict of Interest}

None declared.

\section{Acknowledgments}

The authors want to acknowledge useful inputs from Dr. Anchal Aggarwal and Dr. Shikha Chawla, who are both serving as consultants, and Christy Alekchander and Anjali Sharma, who are both serving as medical physicists in the Department of Radiation Oncology at the Patel Hospital in Jalandhar, Punjab, India.

\section{References}

1 Vargo JA, Beriwal S. Image-based brachytherapy for cervical cancer. World J Clin Oncol 2014;5(5):921-930

2 Mahantshetty U, Khanna N, Swamidas J, et al. Trans-abdominal ultrasound (US) and magnetic resonance imaging (MRI) correlation for conformal intracavitary brachytherapy in carcinoma of the uterine cervix. Radiother Oncol 2012;102(1):130-134

3 Spartacus RK, Dana R, Gaur P, Nandwana U, Rastogi K, Agarwal $\mathrm{N}$. The role of ultrasound-guided applicator placement in intracavitary brachytherapy for cervical cancer. J Med Soc 2018;32:7-11
Address for correspondence Sajal Goel, DNB, Department of Radiation Oncology, Patel Hospital, Civil Lines, Jalandhar 144001, Punjab, India (e-mail: drsajal.goel@gmail.com).
DOI https://doi.org/ 10.1055/s-0039-3401473 ISSN 2454-6798.
C2019 Spring Hope Cancer Foundation \& Young Oncologist Group of Asia
License terms

$($ () (1) $\Theta \circledast$ 
4 ICRU. Prescribing, recording, and reporting brachytherapy for cancer of cervix: Report 89. Journal of the International Commission on Radiation Units and Measurements 2013;13(1):1-258

5 Bandyopadhyay A, Basu P, Roy K, Das S, Banerjee S. Treatment of locally advanced carcinoma cervix with special emphasis on brachytherapy: A practice pattern survey among young radiation oncologist of India. South Asian J Cancer 2018;7(4):231-235

6 Pötter R, Georg P, Dimopoulos JC, et al. Clinical outcome of protocol based image (MRI) guided adaptive brachytherapy combined with 3D conformal radiotherapy with or without chemotherapy in patients with locally advanced cervical cancer. Radiother Oncol 2011;100(1):116-123
7 Mahantshetty U, Krishnatry R, Hande V, et al. Magnetic resonance image guided adaptive brachytherapy in locally advanced cervical cancer: an experience from a tertiary cancer center in a low and middle income countries setting. Int J Radiat Oncol Biol Phys 2017;99(3):608-617

8 Chakraborty S, Mahantshetty U, Chopra S, et al. Income generated by women treated with magnetic resonance imaging-based brachytherapy: a simulation study evaluating the macroeconomic benefits of implementing a high-end technology in a public sector healthcare setting. Brachytherapy 2017;16(5):981-987 